\title{
Editorial
}

\section{Nursing Home Dilemmas}

\author{
Lindsay E. Nicolle, MD
}

This issue offers two contributions to our expanding understanding of infections and antimicrobial resistance in the nursing home population. ${ }^{1,2}$ At any time, a greater number of North Americans are institutionalized in long-term-care facilities, primarily nursing homes, than in acute-care facilities, Despite this, until relatively recently, the burden of infections in this population and the complex and confusing issues of diagnosis and appropriate therapy have been relatively neglected. Notably, this journal has been a leader in a critical analysis of this field through the articles published over the past 8 years as "Topics in Long-Term Care" under the editorship of David Bentley, MD. There is now a wider and increasing interest in issues related to infection in the nursing home. The heightened profile partly may reflect the aging of our populations, but seems driven more by recognition of the nursing home as a cauldron of bacterial colonization and infection, antibiotic therapy, and antimicrobial-resistant organisms. ${ }^{3}$ The current societal focus on the potential catastrophes of antimicrobial resistance has raised the profile of the nursing home.

What do we know about antimicrobial resistance in nursing homes? The prevalence of colonization with antimicrobial-resistant organisms, including methicillin-resistant Staphylococcus aureus (MRSA), vancomycin-resistant enterococci (VRE), and some gram-negative organisms, particularly Providencia stuartii, is high in many. ${ }^{3}$ In particular, antimicrobial-resistant organisms likely occur with greater frequency in Veterans' Affairs facilities. ${ }^{4}$ A consistent observation is that underlying patient characteristics are associated more strongly with the presence of resistant organisms in a given patient than are measures of antimicrobial use. Thus, highly debilitated patients characterized by the presence of decubitus ulcers, gastrostomy feeding tubes, and tracheostomies repeatedly have been identified as most likely harboring resistant organisms. Clinical variables consistent with increased debility are predictors of colonization or infection with antimicrobialresistant organisms in a given resident. The study by Muder et al $^{1}$ confirms, again, the preeminent importance of resident characteristics, in this case for resistant Enterbacteriaceae identified through the clinical laboratory. We also know that antimicrobialresistant organisms, once introduced, tend to persist within the individual and the facility. Antimicrobial pressure from intensive, frequently broad-spectrum, antimicrobial use has some role in this persistence, as the study of Muder et al reports for Pseudomonas aeruginosa clinical isolates.

We also know that patients in long-term-care facilities frequently are transferred to acutecare facilities. The antimicrobial-resistant organisms present in the long-term-care facility are carried with the patient to the acute-care facility and occasionally may be transmitted to other patients in the acute-care facility Transport of resistant organisms in the opposite direction, ie, from the acute-care facility to the longterm-care facility, also is frequent. The evidence from the experience with MRSA and VRE is incomplete, but suggestive that these organisms originated in acute-care facilities and were introduced from these facilities into nursing homes. The nursing home is an

From the Health Sciences Center, Winnipeg, Manitoba, Canada.

Address reprint requests to Lindsay $E$. Nicolle, MD, FRCPC, Health Sciences Centre, Department of Medicine, GC430-820 Sherbrook St, Winnipeg, Manitoba R3A 1R9, Canada; e-mail: nicolle@cc.umanitoba.ca.

9\%ED-115. Nicolle LE. Nursing home dilemmas. Infect Control Hosp Epidemiol 1997;18:806-808. 
effective amplifier of antimicrobial-resistant organisms because of the clinical characteristics of patients and the intense antimicrobial use, but is likely seldom the de novo originator of these resistant strains. Nonetheless, efforts by nursing homes to prevent antimicrobial resistance by refusing admission to patients known to be colonized or infected have not been shown to be an appropriate approach to avoid the importation of resistant organisms.

What do we not know about antimicrobialresistant organisms in long-term-care facilities? The most disconcerting deficiency in our information is the limited understanding of the impact of resistant bacteria on individuals resident in long-term-care facilities, on the long-term-care facility itself, and, in fact, in our society generally. Resistant organisms commonly are isolated as colonizing flora of the upper respiratory tract and skin and wound surfaces, or from asymptomatic bacteriuria. Limited information relevant to MRSA suggests morbidity from infection in a long-term-care facility did not increase with introduction of MRSA. ${ }^{5}$ The only infection that is, quantitatively, an important contributor to mortality in nursing homes is pneumonia. ${ }^{3}$ Many nursing home residents who die with pneumonia are receiving comfort care, and death from pneumonia may be a desired outcome rather than a failure of therapy. While currently there is no evidence to support increased mortality directly related to increased prevalence of resistant organisms, the information available is too limited. Recent reports of outbreaks caused by resistant pneumococci are a concern. ${ }^{6}$ Overall, however, evidence does not suggest increased patient morbidity or mortality attributable to what, in some institutions, is a large burden of resistant organisms.

One area where resistant organisms certainly may have an impact on both the resident and the facility is in isolation strategies used for colonized or infected patients. An approach to isolation that incorporates the relatively strict barrier approaches of the acute-care facility certainly will have a negative impact on the patient and on the facility. The social and rehabilitative aspects of care for long-term-care facility residents necessitate communal interactions. Limitations in care due to aggressive isolation will impair quality of life and, frequently, functional status. The high likelihood of long-term carriage of resistant organisms means some nursing home residents virtually are sentenced to a lifetime of isolation in single rooms cared for by fully barriered attendants if the approaches appropriate for acute-care facilities are instituted. In the absence of evidence that a resident colonized or infected with a resistant organism is harmful to other residents, restrictions in activities are not justified currently. Fortunately, it is becoming more widely understood that managing patients with resistant organisms in long-term-care facilities requires an understanding of the unique features of long-term-care facilities and their patients.? Some important questions include the following: What is the impact of resistant organisms on the quality of life of the long-term-care facility resident? What is the impact of resistant organisms on rehabilitation in such individuals? These are questions that must be answered if we are to manage antimicrobial resistance in long-term-care facilities appropriately.

What else do we not know? Infections are exceedingly common in residents of nursing homes, and many of these infections legitimately require antimicrobial therapy. ${ }^{3}$ The increasing acuity and use of invasive devices in nursing home residents resulting from the more rapid transfer of residents out of acute-care facilities further increases the high frequency of infection. With the high degree of patient debility, opportunities for transmission of organisms between patients inherent in the institutional setting, the high frequency of infections with valid indications for antimicrobial therapy, and the frequency of asymptomatic bacteriuria and mucosal colonization with potential pathogens, how feasible is it to prevent antimicrobial resistance in the nursing home setting? While antimicrobials frequently are used inappropriately in the nursing home, there would be intense exposure to antimicrobials in this environment even if they were used optimally. ${ }^{8}$ Given the uncertainties in clinical and laboratory diagnostic approaches, a more appropriate use of antimicrobials may require decisions not to use antimicrobials in clinical scenarios which, in any other population, would be considered indications for antimicrobial therapy. We probably are not yet ready for this. Again, we return to the issue of more clearly understanding the effects of antimicrobial resistance, and using this information to frame our approaches to management of antimicrobial resistance and antimicrobial use, rather than simply the presence of resistant organisms.

So what can we do with this problem of antimicrobial resistance in long-term-care facilities? Efforts to control infection or transmission of clinically significant organisms between patients are essential. The Society for Healthcare Epidemiology of America and the Association for Professionals in Infection Control and Epidemiology guidelines published here provide a rational framework for the long-term-care facility. ${ }^{2}$ This document focuses on current knowledge specific to long-term-care facilities. It acknowledges the needs of the long-term-care resident and 
the limitations in resources and expertise that are, frequently, a characteristic of nursing homes. It also clearly highlights the paucity of relevant clinical studies available to date in this population. Such studies will be essential not only to evaluate strategies for infection control but also to clarify strategies for optimizing antimicrobial use in these populations.

We can conclude that antimicrobial-resistant organisms are common in some nursing homes, and infections are frequent in all. If we are to use this information to further our approach to the problem of antimicrobial resistance, we must answer additional questions. Studies to measure the effects of antimicrobial resistance in the long-term setting are essential, as are studies to determine what, if anything, may limit resistance and its impact. These studies must be relevant to this population, particularly in acknowledging the imprecision in clinical and laboratory diagnosis inherent in the long-term-care facility population. They should reflect experience in multiple institutions so we can begin to understand to what degree observations are institution-specific and to what extent generalizable. The current interest in antimicrobial resistance provides an opportunity to advance our awareness of issues related to nursing homes. We owe it to our society, and especially to its senior members, to address these issues rationally and from the perspective that the long-term-care facility is different than the acute-care facility, although antimicrobial-resistant organisms are common to both.

\section{R E F E R E N C E S}

1. Muder RR, Brennen C, Drenning SD, Stout JE, Wagener MM. Multiply antibiotic-resistant gram-negative bacilli in a longterm-care facility: a case-control study of patient risk factors and prior antibiotic use. Infect Control Hosp Epidemiol 1997:18:809-813.

2. Smith PW, Rusnak PG. Infection prevention and control in the long-term-care facility. Infect Control Hosp Epidemiol 1997:18:831-849.

3. Nicolle LE, Strausbaugh LJ, Garibaldi RA. Infections and antibiotic resistance in nursing homes. Clin Microbiol Rev 1996;9:1 17.

4. Mulhausen PL, Harrell LJ, Weinberger M, Kochersberger GG, Feussner JR Contrasting methicillin-resistant Staphylococcus aureus colonization in Veterans' Affairs and community nursing homes. Am J Med 1996;100:24-31.

5. Strausbaugh LJ, Jacobson C, Sewell DL, Potter S, Ward TT Methicillin-resistant Staphylococcus aureus in extended care facilities: experiences in a Veterans' Affairs nursing home and a review of the literature. Infect Control Hosp Epidemiol 1991;12:36-45.

6. Centers for Disease Control and Prevention. Outbreaks of pneumococcal pneumonia among unvaccinated residents in chronic care facilities. MMWR 1997:46:60-62.

7. Strausbaugh LJ, Crossley KB, Nurse BA, Thrupp LD, SHEA Long-Term-Care Committee. Antimicrobial resistance in long term care facilities. Infect Control Hosp Epidemiol 1996;17:120129.

8. Nicolle LE, Bentley D, Garibaldi R, Neuhaus E, Smith P, SHEA Long-Term-Care Committee. Antimicrobial use in long-termcare facilities. Infect Control Hosp Epidemiol 1996;17:119-128.

\section{Corrections}

\section{Primary Prevention and Rubella Immunity: Overlooked Issues in the Outpatient Obstetric Setting}

In the September issue of Infection Control and Hospital Epidemiology, there were two errors in the article "Primary Prevention and Rubella Immunity: Overlooked Issues in the Outpatient Obstetric Setting" (1997;18:633-636).

Page 634, column 2, line 23, should have read "Respondents from states with legal requirements for rubella immunity had a sigficicantly higher rate of self-reported immunity compared to physicians from states without such laws $(90.5 \%$ vs $88.7 \%$, respectively; OR, 2.58; 95\% confidence interval $\left.\left[\mathrm{CI}_{95}\right], 1.70-3.92\right)$."

Page 635, column line 1, line 36, should have read "The higher rate of immunity (90.5\% vs $88.7 \%$ ) among physicians in states with legal require- ments suggests that enacting legislation may improve rubella immune status among practicing physicians, but this should be interpreted with caution, because there is insufficient evidence that a legal requirement will assure immunity"

We apologize for any inconvenience these errors may have caused our readers.

\section{A Cluster of Serious Escherichia coli Infections in a Neonatal Intensive-Care Unit}

In the November issue of Infection Control and Hospital Epidemiology, an error was introduced during the final stages of production in the article "A Cluster of Serious Escherichia coli Infections in a
Neonatal Intensive-Care Unit" (1997; 18:774-776). The last sentence on page 774 should have read "This baby was in the intermediate nursery with the twins for 3 weeks before his cultures became positive for $E$ coli, and for 11 days his bed was within $5 \mathrm{ft}$ of theirs."

We apologize for any inconvenience the error may have caused our readers. 